\title{
On the mod-2 cohomology of some 2-Postnikov towers
}

\author{
Nguyễn Thế Cường * \\ Lionel Schwartz ${ }^{\dagger}$
}

May 20, 2020

\begin{abstract}
The present note presents some results about the mod-2 cohomology, modulo nilpotent elements elements of the fiber $E$ of a decomposable map $\psi: K(\mathbb{Z}, 2) \rightarrow K(\mathbb{Z} / 2, p)$. This is more an announcement and a brief description of the tools that are used: Lannes' $T$ functor and the Eilenberg-Moore spectral sequence.
\end{abstract}

\section{Introduction}

Let $E$ be the homotopy fiber of a map $\psi: K\left(\mathbb{F}_{2}, q\right) \rightarrow K\left(\mathbb{F}_{2}, p\right)$ between two Eilenberg-Mac Lane spaces, the homotopy class $[\psi]$ belongs to $\mathrm{H}^{q}\left(K\left(\mathbb{F}_{2}, q\right), \mathbb{F}_{2}\right)$. If $\psi$ is a stable map the work of Larry Smith, David Kraines [22] 23] [10] and others describes the mod-2 cohomology of $E$. The results of L. Smith concern the fiber of an $H$-map between $H$-spaces. Those of Kraines concern more specific examples. One of their main tool is the Eilenberg-Moore spectral sequence.

To make notation shorter we will write $K_{n}$ for the Eilenberg-Mac Lane space $K\left(\mathbb{F}_{2}, n\right)$ in all the sequel. In all this note the mod-2 singular cohomology of a space $X$ will be denoted by $\mathrm{H}^{*}(X)$.

*Nguyễn Thế Cường, Falculty of Mathematics, Mechanics and Informatics, VNU University of Science, 334 Nguyễn Trãi, Thanh Xuân, Hà Nôi, Viêt Nam

${ }^{\dagger}$ Lionel Schwartz, LAGA, UMR 7539 et LIA Formath Vietnam du CNRS, Université Paris Nord Av. J. B. Clément 93430 Villetaneuse, France 
In this note we study the case where (the homotopy class of) $\psi: X \rightarrow K_{p}$ is a decomposable element in the cohomlogy group $\mathrm{H}^{p}(X): \psi=\sum_{i} a_{i} b_{i}$, with $\left|a_{i}\right|>0,\left|b_{i}\right|>0$. One investigates what is possible to say on the cohomology of the homotopy fiber modulo the ideal of nilpotent elements.

After general results one focuses on the case $X=K_{q}$.

The main statements are:

Theorem 1.1. There is a monomorphism:

$$
\frac{\left(\mathrm{H}^{*}(X) /\left(\psi^{*}\left(\iota_{p}\right)\right)\right.}{N i l} \hookrightarrow \frac{\mathrm{H}^{*}(E)}{N i l}
$$

where Nil denotes the ideal of nilpotents elements.

This a reformulation of of [13, Théorème 0.5]

Theorem 1.2. There is an epimorphism, modulo nilpotent elements:

$$
\mathrm{H}^{*}(E) \rightarrow \mathrm{H}^{*}\left(K_{p-1}\right)
$$

This means that for any element $x \in \mathrm{H}^{*}\left(K_{p-1}\right)$ some power $x^{2^{h}}$, $h$ large enough, is in the image.

Part of the results are to some extent, already contained in [19] and [20], but are made here more precise. The next questions are to decide how these two theorems "match" together and to describe the right hand side quotient more explicitly in some cases. As we have said we are going to discuss this modulo nilpotent elements.

The techniques described in the note makes the following conjecture very plausible. The notations are explained after the statement.

Conjecture 1.3. (Weak form) As graded algebras one has:

$$
\sqrt{\mathrm{H}^{*}(E)} \cong \sqrt{\mathrm{H}^{*}(X) /\left(\psi^{*}\left(\iota_{p}\right)\right)} \otimes \mathrm{H}^{*}\left(K_{p-1}\right)
$$

Conjecture 1.4. (Strong form) As unstable algebras one has:

$$
\sqrt{\mathrm{H}^{*}(E)} \cong \sqrt{\mathrm{H}^{*}(X) /\left(\psi^{*}\left(\iota_{p}\right)\right)} \otimes \mathrm{H}^{*}\left(K_{p-1}\right)
$$

The algebra $\sqrt{\mathrm{H}^{*}(Y)}$, for any space $Y$, denotes the quadratic closure of the quotient of the (graded) algebra $\mathrm{H}^{*}(Y)$ by the ideal of nilpotent elements $N i l$. The unstable algebra $\sqrt{\mathrm{H}^{*}(Y)}$ is the smallest (graded) algebraic extension, in the sense of J. F. Adams and C. Wilkerson [1, of $\mathrm{H}^{*}(Y) / N i l$ which contains all square roots.

For example:

- $\sqrt{\mathrm{H}^{*}\left(K_{p}\right)}=\mathrm{H}^{*}\left(K_{p}\right)$; 
- consider $\mathrm{H}^{*}\left(\mathbb{C} P^{\infty}\right) \cong \mathbb{F}_{2}\left[u^{2}\right]$ then $\sqrt{\mathrm{H}^{*}\left(\mathbb{C} P^{\infty}\right)}=\mathrm{H}^{*}\left(\mathbb{R} P^{\infty}\right)=\mathbb{F}_{2}[u]$;

- $\sqrt{K} \cong \mathbb{F}_{2}$ for all finite connected unstable algebra $K$.

From now on we discuss examples.

The mod-2 cohomology of an elementary abelian 2-group $V_{n}$ of rank $n$ denoted by $\mathrm{H}^{*} V_{n}$, is a graded polynomial algebra on $n$ generators of degree $1, \mathbb{F}_{2}\left[x_{1}, \ldots, x_{n}\right]$, these generators may also be denoted also by $u, v, w, \ldots$.

Let $X$ be the Eilenberg-Mac Lane space $K_{2}$. Let $Q_{i}, i \geq 0$ be the Milnor operation of degree $2^{i+1}-1$ [15]. One has [21]:

$$
\mathrm{H}^{*}\left(K_{2}\right) \cong \mathbb{F}_{2}\left[\iota_{2}, Q_{0}\left(\iota_{2}\right), Q_{1}\left(\iota_{2}\right), \ldots, Q_{i}\left(\iota_{2}\right), \ldots\right] \quad .
$$

We now describe the right hand side algebra $\mathrm{H}^{*}\left(K_{2}\right) /\left(\psi^{*}\left(\iota_{p}\right)\right)$.

Proposition 1.5. If $[\psi]=\iota_{2}^{t} \in \mathrm{H}^{*}\left(K_{2}\right), t>1$, then

$$
\sqrt{\mathrm{H}^{*}\left(K_{2}\right) \otimes_{\mathrm{H}^{*} K_{p}} \mathbb{F}_{2}} \cong \mathbb{F}_{2}
$$

Proposition 1.6. If $[\psi] \in \mathrm{H}^{*}\left(K_{2}\right)$ is a nontrivial monomial in the polynomial generators of $\mathrm{H}^{*}\left(K_{2}\right)$, involving at least one $Q_{i}\left(\iota_{2}\right)$, then

$$
\sqrt{\mathrm{H}^{*}\left(K_{2}\right) \otimes_{\mathrm{H}^{*} K_{p}} \mathbb{F}_{2}} \cong \mathrm{H}^{*}(B \mathbb{Z} / 2) \cong \mathbb{F}_{2}[u]
$$

Proposition 1.7. If $[\psi]=\alpha d_{2}$, with $d_{2}=\iota_{2} Q_{0}\left(\iota_{2}\right)+Q_{1}\left(\iota_{2}\right)$, $\alpha$ any nontrivial monomial, then

$$
\sqrt{\mathrm{H}^{*}\left(K_{2}\right) \otimes_{\mathrm{H}^{*} K_{p}} \mathbb{F}_{2}} \cong D(2)
$$

where $D(2)=\mathrm{H}^{*}\left(V_{2}\right)^{\mathrm{GL}_{2}\left(\mathbb{F}_{2}\right)}$ is the mod-2 Dickson algebra.

Proposition 1.8. If $[\psi]=\alpha h_{2}$ with $h_{2}=\iota_{2}^{2} Q_{1}\left(\iota_{2}\right)+Q_{0}\left(\iota_{2}\right)^{3}+\iota_{2}^{3} Q_{0} \iota_{2}, \alpha$ any nontrivial monomial, then

$$
\sqrt{\mathrm{H}^{*}\left(K_{2}\right) \otimes_{\mathrm{H}^{*} K_{p}} \mathbb{F}_{2}} \cong H_{2}
$$

where $H_{2} \subset \mathrm{H}^{*}(B(\mathbb{Z} / 2 \times \mathbb{Z} / 2)) \cong \mathbb{F}_{2}[u, v],|u|=|v|=1$ is the unstable subalgebra generated by

$$
\left\{u v, u v(u+v), u v\left(u^{3}+v^{3}\right), \ldots, u v\left(u^{2^{n}-1}+v^{2^{n}-1}\right), \ldots\right\} \quad .
$$

This is also the subalgebra of $\mathbb{F}_{2}[u, v]$ generated by

$$
w_{2}, w_{1} w_{2}, w_{1}^{2} w_{2}, \ldots, w_{1}^{n} w_{2}, \ldots \text { where }
$$

$w_{1}, w_{2}$ are the universal Stiefel-Whitney classes. 
Proposition 1.9. If $[\psi]=\alpha d_{2} h_{2}, \alpha$ any monomial, then

$$
\sqrt{\mathrm{H}^{*}\left(K_{2}\right) \otimes_{\mathrm{H}^{*} K_{p}} \mathbb{F}_{2}} \cong \sqrt{M_{2}}
$$

where $M_{2} \subset \mathrm{H}^{*}(B(\mathbb{Z} / 2 \times \mathbb{Z} / 2))^{\times 2} \cong \mathbb{F}_{2}[u, v]^{\times 2}$, is the unstable subalgebra which is the fiber product of $D(2)$ and $H_{2}$ over $\mathbb{F}_{2}[u]$ over the nontrivial morphisms $D(2) \rightarrow \mathbb{F}_{2}[u]$ and $H_{2} \rightarrow \mathbb{F}_{2}[u]$.

In fact all of the preceding results (1.5 to 1.8) hold without the assumption "nontrivial momomial". This hypothesis is used in the sequel.

Exercice 1.10. Find classes $[\psi] \in \mathrm{H}^{p}\left(K_{2}\right)$ such that $\sqrt{\mathrm{H}^{*}\left(K_{2}\right) /\left(\psi^{*}\left(\iota_{p}\right)\right.}$ is the unstable subalgebra of $\mathbb{F}_{2}[u, v, w]$ generated by $u v+w^{2}$.

Proposition 1.11. In all of these examples the weak form of the conjecture olds. The strong form also holds except in Proposition 1.9, in which case it is true if $p-1$ is odd.

We will use the T-technology of J. Lannes [11, [20] and also the EilenbergMoore spectral sequence [18], 23]. We make the necessary recollections in Section 1. In Section 2 we discuss the right hand side quotients in 3.3. In Section 3 we prove Theorems 1.1 and 1.2, In Section 4 we prove 1.11. The methods developed is very much likely to extend to solve Conjecture 1.3.

Tihis work will be continued in order to generalize the results, in two directions. The first direction is to extend the results on the reduced part of the cohomology to some more general examples. The second one (and this is the reason for Theorem 4.5) is to extend the results to the 1-nilpotent part of the cohomology. It would be also worth, harder but possible, to try to get some informations about the space of maps $\operatorname{map}\left(B V \wedge \mathbb{R} P^{2}, E\right)$, this would give insights on the 2-nilpotent part of the cohomology of $E$.

Acknowledgement: This work was initiated originally during the first months of the PhD thesis of the first author who made a talk about it at the congress of the learned societies in mathematics of France and Vietnam in Hué (2012). It started again and grew while the second author was visiting VIASM in Hanoï in November-December 2019. This research is funded by the Vietnam National University, Hanoi (VNU) under project number QG.20.28.

\section{Recollections about unstable modules and algebras.}

We recall here some notations and facts about unstable modules and unstable algebras over the Steenrod algebra. 
One denotes as usual $\mathcal{U}$ the abelian category of unstable modules over the Steenrod algebra $\mathcal{A}_{2}$, and by $\mathcal{K}$ the category of unstable algebras. An unstable module $M$ is a (graded) module over the (graded) Steenrod algebra which satisfies $\operatorname{Sq}^{i}(m)=0$ for any $m \in M$ such that $|m|<i$. An unstable algebra is a (graded) algebra which is also an object in $\mathcal{U}$, the two structures being compatible : the Cartan formula and the restriction axiom hold.

As usual, $\Sigma$ denotes the suspension functor in the category $\mathcal{U}$. An unstable module $M$ is a suspension if and only if the map $\mathrm{Sq}_{0}: M \rightarrow M$, $m \mapsto \mathrm{Sq}^{|m|}(m)$ is trivial. Let $\mathcal{N} i l_{i}$ be the Serre classe in $\mathcal{U}$ generated by $i$-th suspensions : it is closed by sub-objects, quotients, extensions and colimits [19], 20]; $\mathcal{N} i l_{1}$ is more commonly denoted by $\mathcal{N} i l$. In an unstable algebra $K$ the ideal consisting of all nilpotent elements is stable under the action of the Steenrod algebra, and is the largest submodule of $K$ in $\mathcal{N} i l$.

Details can be found, for example, in [24], [11], [19], [14], [5].

We will say that a morphism $f: M \rightarrow N$ of unstable modules is an $F$-monomorphism (resp. epimorhism) if the kernel (resp. cokernel) are in $\mathcal{N} i l$. The definition restricts (and is more classical) to unstable algebras: $f: K \rightarrow L$, is an $F$-monomorphism if any element in the kernel is nilpotent, and an $F$-epimorphism if for any $x \in L$ there exists $n$ such that $x^{2^{n}}$ is in the image.

Being an $F$-monomorphism and an $F$-epimorphism at the same time is called an $F$-isomorphism.

As the category $\mathcal{N} i l$ is a localizing subcategory [7], in the quotient category $\mathcal{U} / \mathcal{N} i l$ an $F$-isomorphism becomes an isomorphism. The canonical functor $\mathcal{U} \rightarrow \mathcal{U} / \mathcal{N} i l$ has a right adjoint and there is a localisation functor $\ell: \mathcal{U} \rightarrow \mathcal{U}$. The unstable module $\ell(M)$ is $\mathcal{N}$ il-closed [7]. If $K$ is an unstable algebra, then so is $\ell(K)$. Moreover the unit of the adjunction $\mathcal{K}: K \rightarrow \ell(K)$ is a morphism of unstable algebras [2].

The ideal of nilpotent elements in $\ell(K)$ is trivial and $\ell(K)$ is quadratically closed. This last property means that one cannot find $J \supset \ell(K)$, an element $x \in J \backslash \ell(K)$, with $x^{2} \in \ell(K) \backslash\{0\}$ [14] For this reason we are going to denote $\ell(K)$ by $\sqrt{K}$.

Now, recall the functor $f: \mathcal{U} \rightarrow \mathcal{F}$, where $\mathcal{F}$ is the category of functors from the category of finite dimensional $\mathbb{F}_{2^{-}}$vector spaces to the category of $\mathbb{F}_{2^{-}}$vector spaces. The functor $f$ sends an unstable module $M$ to the functor $V \mapsto \operatorname{Hom}_{\mathcal{U}}\left(M, \mathrm{H}^{*}(V)\right)^{\#}$. It is shown in [9] that $f$ induces an equivalence (also denoted by $f$ ) $f: \mathcal{U} / \mathcal{N} i l \rightarrow \mathcal{F}_{\omega}$ of $\mathcal{U} / \mathcal{N} i l$ with the subcategory of analytic functors $\mathcal{F}_{\omega}$.

The dual considered above is a continuous one. Indeed, $\operatorname{Hom}_{\mathcal{U}}\left(M, \mathrm{H}^{*}(V)\right)$ has a natural profinite structure ([9]). However in the cases we consider all vector spaces are finite dimensional. Thus we will ignore profinite structures.

Recall that Lannes' functor $T_{V}: \mathcal{U} \rightarrow \mathcal{U}$, left adjoint to $M \mapsto \mathrm{H}^{*}(V) \otimes M$, 
is exact and commute with tensor product [11]. Following [9] $f(M)(V)$ is defined to be $T_{V}(M)^{0}$.

As in (9]), let $g: \mathcal{K} \rightarrow \mathcal{G}$, where $\mathcal{G}$ is the category of contravariant functors from the category of finite dimensional $\mathbb{F}_{2}$-vector spaces to the category of sets, be defined by $K \mapsto\left\{V \mapsto \operatorname{Hom}_{\mathcal{K}}\left(K, \mathrm{H}^{*}(V)\right)\right\}$. It induces an equivalence (also denoted by $g$ ) $g: \mathcal{K} / \mathcal{N} i l \rightarrow \mathcal{G}_{\omega}$ to the category of set valued analytic functors.

Recall that Lannes' "linearization theorem" writes as:

$$
f(K)(V) \cong \mathbb{F}_{2}^{\operatorname{Hom}_{\mathcal{K}}\left(K, \mathrm{H}^{*}(V)\right)}
$$

where the right hand side denotes the Boolean algebra of set maps from $\operatorname{Hom}_{\mathcal{K}}\left(K, \mathrm{H}^{*}(V)\right)$ to $\mathbb{F}_{2}$.

Again one should need profinite structures on sets, but again here sets are finite.

Let $U: \mathcal{U} \rightarrow \mathcal{K}$ be the Steenrod-Epstein functor: the universal enveloping algebra [20]. Recall (21]) the isomorphism of unstable algebras $\mathrm{H}^{*}\left(K_{n}\right) \cong$ $U(F(n))$ where the free unstable modules $F(n)$ is characterized by

$$
\operatorname{Hom}_{\mathcal{U}}(F(n), M) \cong M^{n}
$$

We will also use later the fact the functor $T_{V}$ commutes with $U: T_{V}(U(M)) \cong$ $U\left(T_{V}(M)\right)$ and the following isomorphisms (which can be deduced rom part 1 of [20] and of [5]):

$$
T_{V}(F(p)) \cong \bigoplus_{0 \leq i \leq p} \Gamma^{i}(V) \otimes F(p-i)
$$

where $\Gamma^{i} \in \mathcal{F}$ is $i$-th divided power functor. And as $\mathrm{H}^{*}\left(K_{p}\right) \cong U(F(p))$ one gets:

$$
T_{V}\left(\mathrm{H}^{*}\left(K_{p}\right)\right) \cong \bigotimes_{0 \leq i \leq p} \mathrm{H}^{*}\left(K\left(\Gamma^{i}\left(V^{\#}\right), p-i\right)\right)
$$

\section{Examples: subfunctors of $S^{2}$ and quo- tients of $\mathrm{H}^{*}\left(K_{2}\right)$}

Next consider the examples alluded to in the introduction:

Example 3.1. The set valued functor $g\left(\mathrm{H}^{*}\left(K_{2}\right)\right)$ :

$$
V \mapsto \operatorname{Hom}_{\mathcal{K}}\left(\mathrm{H}^{*}\left(K_{2}\right), \mathrm{H}^{*} V\right) \cong S^{2}\left(V^{\#}\right)
$$


is filtetered by the rank $r$ of the associated bilinear form (which is an even integer $r=2 k$ ) by subfunctors:

$$
S^{2,0} \subset S^{2,0} \subset S^{2,2} \subset S^{2,4} \subset \ldots S^{2,2 k} \subset \ldots
$$

$A$ subfunctor $G$ is said to be generated in dimension less than $d$ if any element in $G(V)$ is in the image of $f^{*}$, for some linear map $f: V \rightarrow V_{d}$. There is a diagram of subfunctors:

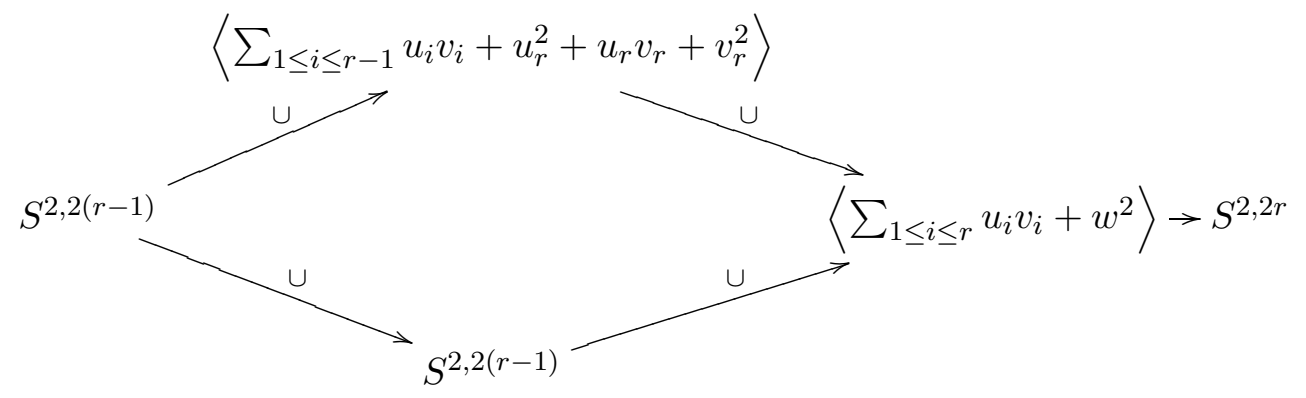

In this diagram quadratic forms generating the subfunctors are written as usual. In rank $2 r$ and dimension $2 r$ there are two non isomorphic forms, one of Arf invariant 1, the other of invariant 0 . In dimension $2 r+1$ and rank $2 r$ there is the form $\left\langle\sum_{1 \leq i \leq r} u_{i} v_{i}+w^{2}\right\rangle$.

Example 3.2. The subfunctors generated in dimension less than 2 are:

- the subfunctor $S^{2,0}$ itself is filtered by the constant subfunctor generated by $S^{*}(0)$, and the subfunctor generated by the form $\left\langle u^{2}\right\rangle \in S^{2}\left(\mathbb{F}_{2}^{\#}\right)$.

- the subfunctor $S^{2,2}$ contains as subfunctor the one generated by the form $\left\langle u^{2}+u v+v^{2}\right\rangle \in S^{2}\left(\left(\mathbb{F}_{2}^{\oplus 2}\right)^{\#}\right)$,

- the one generated by the form $\langle u v\rangle \in S^{2}\left(\left(\mathbb{F}_{2}^{\oplus 2}\right)^{\#}\right)$;

- the subfunctor generated by both forms $\left\langle u v, u^{2}+u v+v^{2}\right\rangle \in S^{2}\left(\left(\mathbb{F}_{2}^{\oplus 2}\right)^{\#}\right)$;

- $S^{2,2}$ is generated by the form $\left\langle u^{2}+u v+v^{2}+w^{2}\right\rangle \in S^{2}\left(\left(\mathbb{F}_{2}^{\oplus 3}\right)^{\#}\right)$.

We come back now to $X=K_{2}$. The quotients (in $\mathcal{K} / \mathcal{N} i l$ ) of $\mathrm{H}^{*}\left(K_{2}\right)$ are classified by subfunctors of $V \mapsto S^{2}\left(V^{\#}\right)$. The correspondance:

$$
\mathcal{K} / \mathcal{N} i l \rightarrow \mathcal{G}_{\omega}, \quad K \mapsto\left\{V \mapsto \operatorname{Hom}_{\mathcal{K}}\left(H, \mathrm{H}^{*}(V)\right)\right\}
$$

is an equivalence of categories. Therefore there is a one-to-one correspondance between subfunctors of $S^{2}(-)^{\#}$ and the quotients in $\mathcal{K} / \mathcal{N} i l$ of $\mathrm{H}^{*}\left(K_{2}\right)$. Recall:

$$
\mathrm{H}^{*}\left(K_{2}\right) \cong \mathbb{F}_{2}\left[\iota_{2}, Q_{0}\left(\iota_{2}\right), Q_{1}\left(\iota_{2}\right), \ldots, Q_{i}\left(\iota_{2}\right), \ldots\right]
$$


We compute explicitly the quotients $\sqrt{\mathrm{H}^{*}\left(K_{2}\right) /\left(\psi^{*}\left(\iota_{p}\right)\right)}$ mentioned in Example 3.2. We do not claim that they are in any sense the best examples and this deserves further investigations.

As $\mathrm{H}^{*}(V) \cong S^{*}\left(V^{\#}\right)$, a (homotopy class of) map: $\psi: K_{p} \rightarrow K_{2}$ determines a natural transformation:

$$
\psi_{*}: S^{2}(-)^{\#} \rightarrow S^{p}(-)^{\#}
$$

It determines a subfunctor in $S^{2}$ as follows: it consists of the set of elements of $S^{2}\left(V^{\#}\right)$ which are sent to 0 by this class:

$$
\left\{s \in S^{2}\left(V^{\#}\right) \mid \psi_{*}(s)=0\right\}
$$

The map $\psi_{*}$ is just a cohomology operation: a polynomial in the Steenrod powers. The computation is done using the usual rules with the Steenrod algebra.

We now illustrate some examples.

- Let $[\psi]$ be $\iota_{2}^{t}$, for some integer $t>1$. In this case

$$
\psi_{*}: S^{2}(-)^{\#} \rightarrow S^{p}(-)^{\#}
$$

is injective. The kernel of this morphism is the constant functor on one point. The corresponding quotient is $\mathbb{F}_{2}$.

- Let $[\psi]$ be $\alpha Q_{i}(\iota)$ for some monomial. As In the case of the subfunctor generated by $\left\langle u^{2}\right\rangle$ any monomial involving one $Q_{i}\left(\iota_{2}\right)$ works because it is zero on $u^{2}$ and non zero on $u v$ and $u^{2}+u v+v^{2}$ :

$$
\begin{gathered}
Q_{i}\left(u^{2}\right)=0 \\
Q_{i}(u v)=u v\left(u^{2^{i+1}-1}+v^{2^{i+1}-1}\right) \\
Q_{i}\left(u^{2}+u v+v^{2}\right)=u v\left(u^{2^{i+1}-1}+v^{2^{i+1}-1}\right)
\end{gathered}
$$

the kernel of $\psi_{*}$ is generated by $\left\langle u^{2}\right\rangle$. The corresponding quotient is therefore $\mathbb{F}_{2}[u]$, whence Proposition 1.6.

- Similarly if $[\psi]=\alpha d_{2}$, and $d_{2}=\left(\iota_{2} Q_{0}\left(\iota_{2}\right)+Q_{1}\left(\iota_{2}\right)\right)$, $\alpha$ any monomial, a direct computation shows that the class $\psi_{*}$ is generated by $\left\langle u^{2}+u v+v^{2}\right\rangle$. The corresponding quotient is the Dickson algebra generated by the Dickson invariants $u^{2}+u v+v^{2}, u v(u+v)$. Proposition 1.7 follows.

The other cases follow. Proposition 3.3 will be proved in section 4 . The subfunctors of Example 3.2 classify (modulo $\mathcal{N} i l$ ) the quotients of transcendance degree at most 2 and 3 of $\mathrm{H}^{*}\left(K_{2}\right), D(2)$ and $H_{2}$ are the only quotients of $\mathrm{H}^{*}\left(K_{2}\right)$ which are integral domain and of transcendance degree 2. This follows from the classification of quadratic forms over $\mathbb{F}_{2}$ (described above) and [9]. 
Proposition 3.3. Let $\psi: K_{2} \rightarrow K_{p}$ be one of the maps in Propositions 1.5 to $1.8 E$ the homotopy fiber of $\psi$, then there is an isomorphism of unstable algebras:

$$
\sqrt{\mathrm{H}^{*}(E)} \cong \sqrt{\mathrm{H}^{*}\left(K_{2}\right) \otimes \mathrm{H}^{*} K_{p} \mathbb{F}_{2}}
$$

For Proposition 1.9 this holds if $p-1$ is odd.

This will be proved in the last section.

\section{Sets of homotopy classes of maps, appli- cations}

Let us start with a classical remark about homotopy classes of maps ([8], Adittionnal topics : Base points and Homotopy 4A).

Proposition 4.1. Let $X$ and $Y$ be two connected pointed $C W$-complexes then the natural map from the set of pointed homotopy classes from $X$ to $Y$ to homotopy classes :

$$
[X, Y]_{*} \rightarrow[X, Y]
$$

is always surjective. It is a bijection if either $X$ is 1-connected or $X$ is $H$-space.

Recall that for an elementary abelian 2-group $V, B V$ is the classifying space of $V$. The fibration $E \rightarrow X \stackrel{\psi}{\rightarrow} K_{p}$ yields a short exact sequence of pointed sets:

$$
\begin{array}{r}
{[\Sigma B V, E]_{*} \rightarrow[\Sigma B V, X]_{*} \rightarrow\left[\Sigma B V, K_{p}\right]_{*} \rightarrow} \\
\rightarrow[B V, E]_{*} \rightarrow[B V, X]_{*} \rightarrow\left[B V, K_{p}\right]_{*}
\end{array}
$$

If $X$ is 1 -connected it is equivalent to :

$$
\begin{array}{r}
{[\Sigma B V, E] \rightarrow[\Sigma B V, X] \rightarrow\left[\Sigma B V, K_{p}\right] \rightarrow} \\
\quad \rightarrow[B V, E] \rightarrow[B V, X] \rightarrow\left[B V, K_{p}\right]
\end{array}
$$

Where:

- The first 3 terms are groups and the sequence is exact in the usual sense.

- The last three terms are pointed sets, the composite morphisms are trivial. 
- Exactness at $[B V, X]_{*}$ means that the (homotopy class) of a map $f$ : $B V \rightarrow X$ lifts to $E$ if and only if the composite $B V \rightarrow X \rightarrow K_{p}$ is trivial.

- Exactness at $[B V, E]_{*}$ means that the (homotopy class) of a map $f$ : $B V \rightarrow E$ lifts to $\left[\Sigma B V, K_{p}\right]$ if and only if the composite $B V \rightarrow E \rightarrow X$ is trivial.

- Moreover $\left[\Sigma B V, K_{p}\right]_{*}$ acts on $[B V, E]_{*}$, and two homotopy classes $f, g$ : $B V \rightarrow E_{d}$ which have the same image in $[B V, X]_{*}$ are in the same orbit under the action of $\left[\Sigma B V, K_{p}\right]$.

- An element of $\left[\Sigma B V, K_{p}\right]$ which acts trivially on $[B V, E]_{*}$ comes from $[\Sigma B V, X]$.

We are going to use the following two theorems:

Theorem 4.2 (11]). Let $X$ be a connected $C W$-complex such that $\mathrm{H}^{*}(X)$ is of finite dimension in any degree and that $\pi_{1}(X)$ is a finite 2-group. Then the natural map

$$
[B V, X] \rightarrow \operatorname{Hom}_{\mathcal{K}}\left(\mathrm{H}^{*}(B V), \mathrm{H}^{*}(X)\right), \quad f \mapsto f^{*}
$$

is a bijection

The second theorem below is outside of the main stream of the note. It is there to show that this type of strategy also allows to get informations on the ideal $N i l \subset \mathrm{H}^{*}(E)$. This will be done done in future articles.

Theorem 4.3. [12] The natural map

$$
[\Sigma B V, X] \rightarrow \operatorname{Hom}_{\mathcal{K}}\left(\mathrm{H}^{*}(X), \mathrm{H}^{*}(\Sigma B V)\right), \quad f \mapsto f^{*}
$$

is a surjection

For an augmented unstable algebra $K$, let $\tilde{K}$ be the ideal of augmentation. Denote by

$$
Q(K):=\frac{\tilde{K}}{\tilde{K} \cdot \tilde{K}}
$$

the unstable module of indecomposable elements. For any unstable algebra $K, Q(K)$ is a suspension. Remark that this is true without connectivity hypothesis on $K$ because $K^{0}$ is a Boolean algebra. One has:

$$
\operatorname{Hom}_{\mathcal{K}}\left(\mathrm{H}^{*}(X), \mathrm{H}^{*}(\Sigma B V)\right) \cong \operatorname{Hom}_{\mathcal{U}}\left(\Sigma^{-1} Q\left(\mathrm{H}^{*}(X)\right), \tilde{\mathrm{H}}^{*}(B V)\right)
$$

Thanks to 4.2 and 4.3 , sequences 4.1 and 4.2 yield the following exact sequences: 


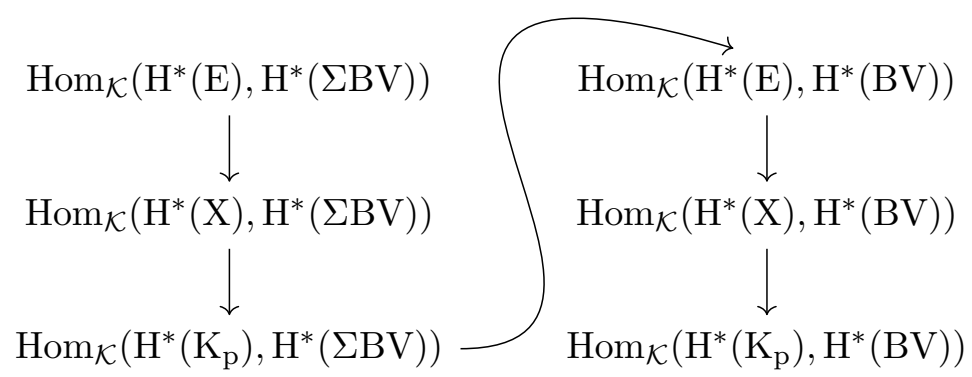

Note that we have the following isomorphisms:

$$
[\Sigma B V, Y]_{*} \cong[B V, \Omega X]_{*} \cong \operatorname{Hom}_{\mathcal{K}}\left(\mathrm{H}^{*}(\Omega X), \mathrm{H}^{*}(B V)\right)
$$

Now we use the hypothesis that the class $\psi$ is decomposable. The following is classical.

Lemma 4.4. If $\psi \in \mathrm{H}^{p}(X)$ is decomposable the class $\Omega \psi \in \mathrm{H}^{p-1}(\Omega X)$ is trivial. Consequently $\Omega E$ is homotopically equivalent to $\Omega X \times K_{p-2}$

Proof. One has a commutative diagram :

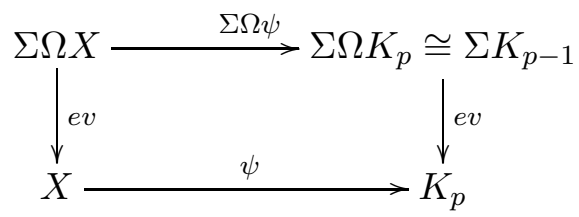

Denote by $\iota_{p}$ the generator of $\mathrm{H}^{p}\left(K_{p}\right)$. The evaluation map ev sends $(t, \omega)$, $t \in[0,1], \omega \in \Omega X$ (resp. $\Omega K_{p}$ ) to $\omega(t)$. As all nontrivial cup-products are trivial in a suspension $\psi \circ e v \in \mathrm{H}^{p}(\Sigma \Omega X)$ is trivial. By commutativity of the diagram $e v \circ \Sigma \Omega \psi$ is trivial. As $e V^{\#}\left(\iota_{p}\right)=\Sigma \iota_{p-1}$ this implies that $\Sigma(\Omega \psi)^{*}\left(\iota_{p-1}\right)=0$, and $(\Omega \psi)^{*}\left(\iota_{p-1}\right)=0$

As $\Omega \psi \sim *$ one gets an inclusion

$$
\operatorname{Hom}_{\mathcal{K}}\left(\mathrm{H}^{*}\left(K_{p-1}\right), \mathrm{H}^{*}(B V)\right) \hookrightarrow \operatorname{Hom}_{\mathcal{K}}\left(\mathrm{H}^{*}(E), \mathrm{H}^{*}(B V)\right)
$$

In other words, we have an injection $g\left(K_{p-1}\right) \rightarrow g\left(\mathrm{H}^{*}(E)\right)$. Because of the equivalence of categories $\mathcal{K} / \mathcal{N} i l \simeq \mathcal{G}_{\omega}$, we get an $F$-epimorphism $\mathrm{H}^{*}(E) \rightarrow \mathrm{H}^{*}\left(K_{p-1}\right)$, whence Theorem 1.2. Thus, $\mathrm{H}^{*}(E) \rightarrow \mathrm{H}^{*}\left(K_{p-1}\right)$ is an $F$-epimorphism: a certain power $\iota_{p-1}^{2^{h}}$ of the fundamental class is in the image of the morphism, however, to find a representative may be hard.

The following result is a consequence of Theorem 4.3, it is mentionned for future investigations: 
Proposition 4.5. Let $E$ be the homotopy fiber of a non homotopically trivial decomposable map $\psi: X \rightarrow K_{p}$. There is a monomorphism of set valued functors:

$$
\left.f\left(\Sigma^{-1} Q\left(\mathrm{H}^{*} E\right)\right) / \mathcal{N} i l\right) \hookrightarrow S^{p-2} \oplus g\left(\mathrm{H}^{*}(\Omega X)\right) \quad .
$$

It follows from 4.3 that one has a surjection:

$$
[\Sigma B V, E] \rightarrow \operatorname{Hom}_{\mathcal{K}}\left(\mathrm{H}^{*}(E), \mathrm{H}^{*}(\Sigma B V)\right) \cong \operatorname{Hom}_{\mathcal{U}}\left(\Sigma^{-1} Q\left(\mathrm{H}^{*}(E), \tilde{\mathrm{H}}^{*} V\right)\right.
$$

as

$$
[\Sigma B V, E] \cong[B V, \Omega E] \cong \operatorname{Hom}_{\mathcal{K}}\left(\mathrm{H}^{*}(\Omega E), H^{*} V\right)
$$

and as we have isomorphisms

$$
\left.\mathrm{H}^{*}(\Omega E) \cong \mathrm{H}^{*}(\Omega X)\right) \otimes \mathrm{H}^{*}\left(K_{p-2}\right)
$$

the inclusion follows.

\section{The Eilenberg-Moore spectral sequence}

We are now going to prove Proposition 3.3. The methods are very much likely to adapt to prove Conjecture 1.3 . We will use the Eilenberg-Moore spectral sequence. Recall various facts about the spectral sequence, references are [18], [23] and [4]. Let $F \rightarrow E \rightarrow B$ be a fiber sequence of pointed spaces, assume that $\pi_{1}(B)$ is a finite 2 -group. The spectral sequence has the following properties :

i. There is a deceasing filtration on $\mathrm{H}^{*}(F)$ :

$$
\ldots \supset F_{-s} \supset \ldots \supset F_{-1} \supset F_{0}
$$

by $\mathcal{A}_{2}$-modules;

ii. The $E_{s, *}^{2}$ page is isomorphic to $\operatorname{Tor}_{\mathrm{H}^{*}(B)}^{-s}\left(H^{*}(E), \mathbb{F}_{2}\right), s \geq 0$;

iii. the differentials are of bidegree $(r, 1-r)$ and are $\mathcal{A}_{2}$-linear;

iv. the spectral sequence is a multiplicative one, the product being given by the shuffle product;

v. it converges towards $\mathrm{H}^{*}(F)$ : the $k$-th term of the graded object associated to the filtration of $\mathrm{H}^{k}(F)$ is $\Sigma^{s} E_{s,-s+k}^{\infty}$;

vi. $\operatorname{Tor}_{\mathrm{H}^{*}(B)}^{-s}\left(H^{*}(E), \mathbb{F}_{2}\right)$ is $(s-1)$-connected and in $\mathcal{N} i l_{s}$ ([20] Theorem 6.4.1).

About the convergence, it is obvious when $B$ is 1-connected, there is a vanishing line of slope 2 : everything below the line $2 u+v=0$ is trivial and 
the filtration of $\mathrm{H}^{*}(F)$ in a given degree is finite. For the general case see [4.

About (vi), $\operatorname{Tor}_{\mathrm{H}^{*}(B)}^{-s}\left(H^{*}(E), \mathbb{F}_{2}\right)$ is not in general an $s$-suspension, even if it is true for $s=1$ and $p=2$ (this is not true if $p>2$ ). Being in $\mathcal{N} i l_{-s}$ follows from the fact that if $M$ is a module over an unstable module $K$, such that the action satisfies the Cartan formula, then $\operatorname{Tor}_{K}^{s}\left(M, \mathbb{F}_{2}\right)$ is $(-s-1)$-connected (see [20] Lemma 6.4.3).

It follows from (ii) and (vi) that $d_{r}\left(E_{s, *}^{2}\right) \in \mathcal{N} i l_{-s-r+1}$.

Applications of these results are given in [19], see for example Theorem 8.7.1 and Proposition 8.7.7.

It is worth and it will be useful to give some more informations on the construction of the spectral sequence. It follows from [18] (see Section 3, the construction of the space $X^{1}$ ) that the -1 step of the filtration on $\mathrm{H}^{*}(F)$ is, up to suspension, the image of $\mathrm{H}^{*}\left(X^{n}\right)$, is defined by the diagram of cofibrations below:

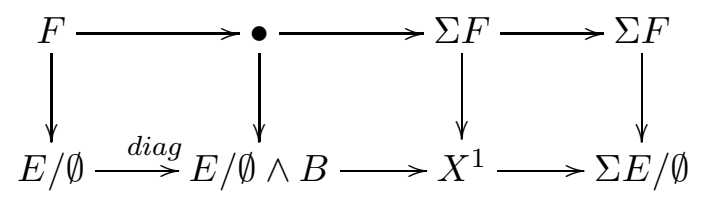

In our case $E=K_{2}$ and $B=K_{p}$.

This works as well when replacing all spaces $Z$ in this diagram by the corresponding mapping $\operatorname{spaces} \operatorname{map}(B V, Z)([3])$.

In order to prove 3.3 one computes the associated graded object of $f\left(\sqrt{\mathrm{H}^{*}(F)}\right)$ with respect to the Eilenberg-Moore filtration. Part (a) of the proposition follows directly. For part (b) one needs to control extensions.

Proposition 5.1. The Eilenberg-Moore filtration on $\mathrm{H}^{*}(F)$ induces a filtration on the functor $f\left(\mathrm{H}^{*} F\right) \cong f\left(\sqrt{\mathrm{H}^{*} F}\right)$. The graded functor associated to this filtration is a (graded) subquotient of the graded functor:

$$
\left\{T_{V}\left(\operatorname{Tor}_{\mathrm{H}^{*}(B)}^{-s}\left(\mathrm{H}^{*}(E), \mathbb{F}_{2}\right)\right)^{s}\right\}_{s \geq 0}
$$

(b) In our cases the spectral sequence degenerates at $E^{2}$. Thus the associated graded functor is equal to the preceding one and isomorphic to:

$$
\left\{\left(\mathbb{F}_{2}^{\Gamma^{2}\left(V^{\#}\right)} \otimes_{\mathbb{F}_{2}^{\Gamma^{p}\left(V^{\#}\right)}} \mathbb{F}_{2}\right) \otimes \Lambda^{s}\left(\Gamma^{p-1}\right)\right\}_{s \geq 0} .
$$

This isomorphism respect the multiplicative structure. In terms of unstable modules this corresponds to:

$$
\left.\sqrt{\mathrm{H}^{*}(X) /\left(\psi^{*}\left(\iota_{p}\right)\right.}\right) \otimes \Lambda^{*}(F(p-1))
$$


We come back to the case of a decomposable map $\psi: K_{2} \rightarrow K_{p}$. One needs to compute: $T_{V}\left(\operatorname{Tor}_{\mathrm{H}^{*}\left(K_{p}\right)}^{-s}\left(\mathrm{H}^{*}\left(K_{2}\right), \mathbb{F}_{2}\right)\right)^{s}$. Recall that: $\operatorname{Tor}_{\mathrm{H}^{*}\left(K_{p}\right)}^{-s}\left(\mathrm{H}^{*}\left(K_{2}\right), \mathbb{F}_{2}\right)$ is the $-s$-th cohomology of the reduced bar complex

$$
\ldots \rightarrow \mathrm{H}^{*}\left(K_{2}\right) \otimes \tilde{\mathrm{H}}^{*}\left(K_{p}\right)^{\otimes s} \rightarrow \mathrm{H}^{*}\left(K_{2}\right) \otimes \tilde{\mathrm{H}}^{*}\left(K_{p}\right)^{\otimes s-1} \rightarrow \ldots \rightarrow \mathrm{H}^{*}\left(K_{2}\right)
$$

As the functor $T_{V}$ is exact and commutes with tensor products, applying it to the sequence 5.1 yields a complex whose $-s$-th cohomology is

$$
\operatorname{Tor}_{T_{V}\left(\mathrm{H}^{*}\left(K_{p}\right)\right)}^{-s}\left(T_{V}\left(\mathrm{H}^{*}\left(K_{2}\right)\right), \mathbb{F}_{2}\right)
$$

(see[20]).

This complex simplifies because $\mathrm{H}^{*}\left(K_{p}\right)$ is an unstable algebra (see the proof of 6.4.3 in [20]). Recall formulae (2.1) and (2.2):

$$
T_{V}(F(p)) \cong \bigoplus_{0 \leq i \leq p} \Gamma^{i}(V) \otimes F(p-i)
$$

and as $\mathrm{H}^{*}\left(K_{p}\right) \cong U(F(p))$ one gets:

$$
T_{V}\left(\mathrm{H}^{*}\left(K_{p}\right)\right) \cong \bigotimes_{0 \leq i \leq p} \mathrm{H}^{*}\left(K\left(\Gamma^{i}\left(V^{\#}\right), p-i\right)\right)
$$

The Boolean of maps from a set $X$ to $\mathbb{F}_{2}$ is denoted by $\mathbb{F}_{2}^{X}, \overline{\mathbb{F}_{2}^{X}}$ denotes the ideal of maps taking the value 0 at a chosen base point; in our case as the sets are vector spaces one chooses 0 as base point.

The evaluation map at the base point $\mathbb{F}_{2}^{X} \rightarrow \mathbb{F}_{2}$ makes $\mathbb{F}_{2}$ a projective module over $\mathbb{F}_{2}^{X}$. As $T_{V}\left(\mathrm{H}^{*}\left(K_{p}\right)\right)^{0} \cong \mathbb{F}_{2}^{\Gamma^{p}\left(V^{\#}\right)}$, it makes

$$
L_{p}=\bigotimes_{1 \leq i \leq p} \mathrm{H}^{*}\left(K\left(p-i, \Gamma^{i}\left(V^{\#}\right)\right)\right.
$$

a projective module over

$$
T_{V}\left(\mathrm{H}^{*}\left(K_{p}\right)\right) \cong \bigotimes_{0 \leq i \leq p} \mathrm{H}^{*}\left(K\left(\Gamma^{i}\left(V^{\#}\right), p-i\right)\right.
$$

Thus:

Lemma 5.2. The reduced bar resolution of $\mathbb{F}_{2}$ over $L_{p}$ is also a projective resolution of $\mathbb{F}_{2}$ over $T_{V}\left(\mathrm{H}^{*}\left(K_{p}\right)\right)$.

Define the unstable algebra $L_{2}$ by $L_{2}=T_{V}\left(\mathrm{H}^{*}\left(K_{2}\right)\right) \otimes_{\mathbb{F}_{2}^{\Gamma^{p}(V \#)}} \mathbb{F}_{2}$. It follows from the preceding lemma that $T_{V}\left(\operatorname{Tor}_{\mathrm{H}^{*}\left(K_{p}\right)}^{-s}\left(\mathrm{H}^{*}\left(K_{2}\right), \mathbb{F}_{2}\right)\right)$ is also the $-s$-th homology of the reduced bar complex:

$$
\cdots \rightarrow L_{2} \otimes \bar{L}_{p}^{\otimes s} \rightarrow L_{2} \otimes \bar{L}_{p}^{\otimes s-1} \rightarrow \cdots \rightarrow L_{2} \rightarrow 0
$$


In degree 0 this is :

$$
\cdots \rightarrow 0 \rightarrow L_{2}^{0} \cong \mathbb{F}_{2}^{\Gamma^{2}\left(V^{\#)}\right.} \otimes_{\mathbb{F}_{2}^{\Gamma^{p}(V \#)}} \mathbb{F}_{2} \rightarrow 0
$$

One has

$$
L_{2} \cong L_{2}^{0} \otimes \mathrm{H}^{*}\left(K_{2}\right) \otimes \mathrm{H}^{*}\left(K\left(V^{\#}, 1\right)\right)
$$

thus

$$
\begin{aligned}
& L_{2}^{1} \cong V^{\sharp} \otimes L_{2}^{0}, \\
& L_{p}^{1} \cong \Gamma^{p-1}\left(V^{\#}\right), \\
& L_{p}^{2} \cong S^{2}\left(\Gamma^{p-1}\left(V^{\#}\right)\right),
\end{aligned}
$$

in degree $s$, place $-s$, Complex (5.2) becomes:

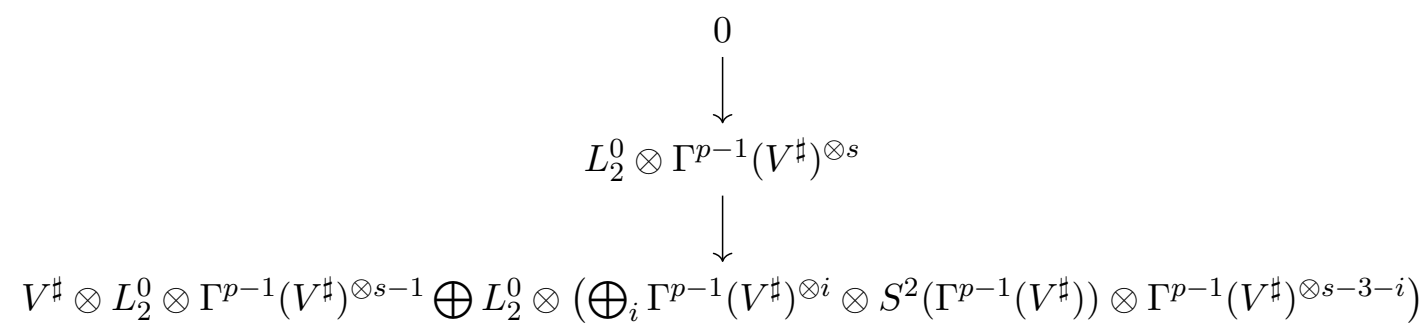

We will show that the morphism is trivial for $s=1$, the map is the one of the module structure. We will show below that the composite:

$$
L_{L_{2}} \otimes \Gamma^{p-1}\left(V^{\#}\right) \hookrightarrow L_{2}^{0} \otimes L_{p}^{1} \otimes V^{\#} \rightarrow L_{2}^{0} \otimes V^{\#}
$$

takes values into $L_{L_{2}^{0}} \otimes V^{\#}$. We will show also it factors through nontrivial the tensor products. As there are no nontrivial morphism from a nontrivial tensor product to $\Gamma^{1}$ (see in [6], " Algèbre de Steenrod, modules instables et foncteurs polynomiaux") the morphism will be shown to be trivial because of the module structure.

It follows, using the multiplicative structure on the bar resolution, that as an algebra, we have a natural isomorphism in $V$ :

$$
\left\{T_{V}\left(\operatorname{Tor}_{\mathrm{H}^{*}\left(K_{p}\right)}^{-s}\left(\mathrm{H}^{*}\left(K_{2}\right), \mathbb{F}_{2}\right)\right)^{s}\right\}_{s \geq 0} \cong\left\{L_{2}^{0} \otimes \Lambda^{s}\left(\Gamma^{p-1}\right)\right\}_{s \geq 0} .
$$

This identifies with the $E^{\infty}$-term on the diagonal $p+q=0$ and therefore is the associated graded of $f\left(\sqrt{\mathrm{H}^{*}(E)}\right), 3.3$ (a) follows. Let us make this a bit more precise: applying the functor $T_{V}$ to the Eilenberg-Moore spectral sequence yields the Eilenberg-Moore spectral sequence for the mapping spaces as observed in [3].

In order to complete the proof one needs to describe how to compute the induced morphism $T_{V}(\psi)$ and gets some informations about the module 
structure of $T_{V}\left(\mathrm{H}^{*}\left(K_{2}\right)\right)$ over $T_{V}\left(\mathrm{H}^{*}\left(K_{p}\right)\right)$. We will make this a bit longer than necessary for future use.

We will show two facts:

- the first one is that $T_{V}(\psi)$ writes as $T_{V}(\psi)^{0} \otimes T_{V}(\psi)^{>0}$,

- the second one is that $T_{V}(\psi)^{1}: \Gamma^{p-1}\left(V^{\#}\right) \otimes F(1)^{1} \rightarrow V^{\#} \otimes F(1)^{1}$ under the hypothesis $\psi$ is decomposable, factors through (a direct sum of) nontrivial tensor products $A \otimes B \otimes F(1)^{1}$. As there are no nontrivial morphisms ([6]) from a nontrivial tensor product $A \otimes B$ to the identity functor we are done.

In the discussion below, we check the first affirmation in all cases: stable classes, sums of classes, products of classes. This is done by inspection. For the second fact one just needs to look at the argument about product of classes.

In degree 0 the morphism is induced by composition of maps: $\psi$ induces a set map, natural in $V, S^{2}\left(V^{\#}\right) \rightarrow S^{p}\left(V^{\#}\right)$. To a (set) map $a: S^{p}\left(V^{\#}\right) \rightarrow \mathbb{F}_{2}$ one associates $a \circ \psi$.

We discuss now the behavior in non zero degrees.

If $\psi$ is a stable class, it is induced by a morphism $F(p) \rightarrow F(2)$ which we will denote also by $\psi$, (i.e. equivalently $\psi=\theta\left(\iota_{2}\right)$ where $\theta$ is a Steenrod operation). The induced morphism $\mathrm{H}^{*}\left(K_{p}\right) \rightarrow \mathrm{H}^{*}\left(K_{2}\right)$ is obtained by applying the Steenrod-Epstein functor $U$ to $\psi$.

The morphism $\left.T_{V}(\psi)\right): T_{V}\left(\mathrm{H}^{*}\left(K_{p}\right) \rightarrow T_{V}\left(\mathrm{H}^{*}\left(K_{2}\right)\right.\right.$ is obtained by applying $U$ to:

$$
T_{V}(F(p)) \cong \bigoplus_{0 \leq i \leq p} \Gamma^{i}(V) \otimes F(p-i) \rightarrow T_{V}(F(2)) \cong \bigoplus_{0 \leq j \leq 2} \Gamma^{j}(V) \otimes F(2-j)
$$

As there are no nontrivial morphisms to (resp. from) $F(0)$ from (resp. to) $F(n)), n>0$, this morphism splits up as a direct sum $T_{V}(\psi)^{0} \oplus T_{V}(\psi)^{>0}$.

Next, as there are no nontrivial morphism from $F(k)$ to $F(\ell)$ if $k<\ell$ the morphism $T_{V}(\psi)^{>0}$ has a triangular matrix in the following sense: the morphisms from $\Gamma^{i}(V) \otimes F(p-i)$ to $\Gamma^{j}(V) \otimes F(2-j)$ can be nontrivial only $p-i \geq 2-j$.

The following steps are to describe the situation on a sums and products of classes.

Let $\psi \in \mathrm{H}^{p}\left(K_{2}\right)$, suppose that $\psi=\alpha+\beta, \alpha \in \mathrm{H}^{p}\left(K_{2}\right), \beta \in \mathrm{H}^{p}\left(K_{2}\right)$. The morphism

$$
T_{V}(\psi): T_{V}\left(\mathrm{H}^{*}\left(K_{p}\right)\right) \rightarrow T_{V}\left(\mathrm{H}^{*}\left(K_{2}\right)\right)
$$

is the composite:

$$
T_{V}\left(\mathrm{H}^{*}\left(K_{p}\right)\right) \rightarrow T_{V}\left(\mathrm{H}^{*}\left(K_{p}\right) \otimes T_{V}\left(\mathrm{H}^{*}\left(K_{p}\right)\right) \stackrel{T_{V}\left(\alpha \otimes T_{V}(\beta)\right.}{\longrightarrow} \cdots\right.
$$




$$
\ldots \stackrel{T_{V}(\alpha) \otimes T_{V}(\beta)}{\longrightarrow} T_{V}\left(\mathrm{H}^{*}\left(K_{2}\right) \otimes T_{V}\left(\mathrm{H}^{*}\left(K_{2}\right)\right) \stackrel{\mu}{\rightarrow} T_{V}\left(\mathrm{H}^{*}\left(K_{2}\right)\right)\right.
$$

where $T_{V}\left(\mathrm{H}^{*}\left(K_{p}\right)\right) \rightarrow T_{V}\left(\mathrm{H}^{*}\left(K_{p}\right) \otimes T_{V}\left(\mathrm{H}^{*}\left(K_{p}\right)\right)\right.$ is obtained by applying $T_{V} \circ U$ to $F(p) \stackrel{\text { diag }}{\longrightarrow} F(p) \oplus F(p)$.

The key step is the one of a product of classes. Let $\psi \in \mathrm{H}^{p}\left(K_{2}\right)$, suppose that $\psi=\alpha \beta, \alpha \in \mathrm{H}^{u}\left(K_{2}\right), \beta \in \mathrm{H}^{v}\left(K_{2}\right), u, v>0$. The morphism

$$
T_{V}(\psi): T_{V}\left(\mathrm{H}^{*}\left(K_{p}\right)\right) \rightarrow T_{V}\left(\mathrm{H}^{*}\left(K_{2}\right)\right)
$$

is the composite:

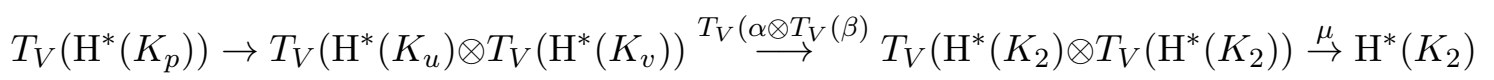

where $T_{V}\left(\mathrm{H}^{*}\left(K_{p}\right)\right) \rightarrow T_{V}\left(\mathrm{H}^{*}\left(K_{u}\right) \otimes T_{V}\left(\mathrm{H}^{*}\left(K_{v}\right)\right)\right.$ is obtained, using the universal property of $U$, from $F(p) \rightarrow F(u) \otimes F(v) \hookrightarrow U(F(u)) \otimes U(F(v))$.

Finally checking each step one gets:

Proposition 5.3. For any class $\psi$ the morphism $T_{V}(\psi)$ is the tensor product of $T_{V}(\psi)^{0}$ and of $T_{V}(\psi)^{>0}$

Corollary 5.4. The morphism $T_{V}(\psi)^{1}$ identifies is the tensor product of $T_{V}(\psi)^{0}$ and $\Gamma^{p-1}\left(V^{\#}\right) \otimes F(1) \rightarrow V^{\#} \otimes F(1)$.

Consider now the induced morphism on $\Gamma^{p-1}\left(V^{\#}\right) \otimes F(1)^{1} \rightarrow V^{\#} \otimes F(1)^{1}$ By construction it factors as:

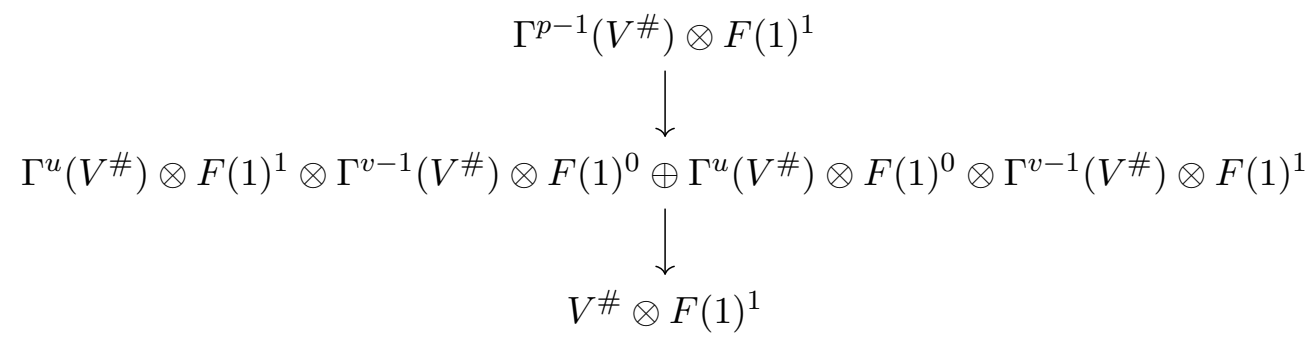

with $u+v=p$. As $u, v>1$ in our cases the second technical result follows.

This proves part (a) of the conjecture.

In order to prove the strong form in 3.3 it is enough to show that the -1 step of the filtration of $f\left(\mathrm{H}^{*}(E)\right), F_{-1}$, is isomorphic to

$$
\tilde{L}_{2}^{0} \otimes \Gamma^{p-1}\left(V^{\#}\right) \oplus \Gamma^{p-1}\left(V^{\#}\right) \oplus L_{2}^{0}
$$

Indeed, if so the direct summand $\Gamma^{p-1}\left(V^{\#}\right)$ generates multiplicatively an exterior algebra $\Lambda^{*}\left(\Gamma^{p-1}\left(V^{\#}\right)\right)$, and thus the graded associated to $\sqrt{\mathrm{H}^{*}(E)}$ 
contains a subfactor $\Lambda^{*}(F(p-1))$ generated on the -1-column of the spectral sequence. The result follows.

Saying it differently it means that a certain power $\iota_{p-1}^{2^{k}}$ is not only in the image of $\mathrm{H}^{*}(E) \rightarrow \mathrm{H}^{*}\left(\mathrm{~K}_{\mathrm{p}-1}\right)$, but that there is a lifting $x$ such that the map

$$
\frac{\mathcal{A}_{2} . x}{N i l} \rightarrow \mathcal{A}_{2} \cdot \iota_{p-1}^{2^{k}} \subset F(p-1)
$$

is an isomorphism.

Example 5.5. Such a lifting may be very hard to find. In the case of the class $\psi=\iota_{2} Q_{1}\left(\iota_{2}\right)$, the class $\mathrm{Sq}^{4} \iota_{5} \otimes 1+\mathrm{Sq}^{2} \iota_{5} \otimes \iota_{2}+\iota_{5} \otimes \iota_{2}^{2}$ is a candidate.

One knows there is a short exact sequence:

$$
0 \rightarrow L_{2}^{0} \rightarrow F_{-1} \rightarrow L_{2}^{0} \otimes \Gamma^{p-1}\left(V^{\#}\right) \cong \Gamma^{p-1}\left(V^{\#}\right) \oplus \overline{L_{2}^{0}} \otimes \Gamma^{p-1}\left(V^{\#}\right) \rightarrow 0
$$

and one shows that it splits.

Because of the multiplicative structure it is enough to show that,

$$
\operatorname{Ext}_{\mathcal{F}}^{1}\left(\Gamma^{p-1}, L_{2}^{0}\right)=0
$$

As is shown below this is true in all the cases we consider, however it is hopeless to expect such a condition to be true in full generality. We will show that the corresponding extension group is non trivial if $p-1$ is odd in the case of some fiber $E$ of a map from $K_{3}$ to $K_{p-1}$.

There is an isomorphism (5] théorème 12.13 page 95$)$ :

$$
\operatorname{Ext}_{\mathcal{F}}^{1}\left(\Gamma^{p-1}, L_{2}^{0}\right) \cong\left(\ell^{1} \circ m\left(L_{2}^{0}\right)\right)^{p-1}
$$

$m$ is the right adjoint of $f, \ell=m \circ f, \ell=m \circ f, \ell$ is left exact and $\ell^{1}$ is the first right derived functor.

The work of G. Powell [16] shows all the of $L_{2}^{0}$ in our list have a finite filtration whose quotients are in the list below. Therefore it is enough to check (5.3) for the functors:

- the constant functor $\mathbb{F}_{2}$,

- $I: V \mapsto \mathbb{F}_{2}^{V \#}$;

- $I_{2}: V \mapsto\left(\mathbb{F}_{2}^{V^{\#}}\right)^{\otimes 2}$;

- their direct summands;

- $B_{2}: V \mapsto\left(\left(\mathbb{F}_{2}^{V^{\#}}\right)^{\otimes 2}\right)^{\mathbb{Z} / 2}$, note that $B_{2} \cong S^{2}(I)$;

- the kernel $\bar{B}_{2}$ of the epimorphism $B_{2} \rightarrow I$;

- $D_{2}: V \mapsto\left(\left(\mathbb{F}_{2}^{V^{\#}}\right)^{\otimes 2}\right)^{\mathrm{GL}_{2}\left(\mathbb{F}_{2}\right)}$; 
- the kernel $\bar{D}_{2}$ of the epimorphism $D_{2} \rightarrow I$.

The first three ones are injective.

For the two following cases, one has $m\left(B_{2}\right) \cong \mathbb{F}_{2}\left[w_{1}, w_{2}\right] \cong \mathbb{F}_{2}[u, v]^{\mathbb{Z} / 2}$. The unstable module $\mathbb{F}_{2}\left[w_{1}, w_{2}\right]$ has an injective resolution which starts like:

$$
\mathbb{F}_{2}\left[w_{1}, w_{2}\right] \hookrightarrow \mathbb{F}_{2}[u, v] \stackrel{1+\tau^{*}}{\rightarrow} \mathbb{F}_{2}[u, v] \stackrel{1+\tau^{*}}{\rightarrow} \mathbb{F}_{2}[u, v] \oplus \mathbb{F}_{2}[u] \rightarrow \ldots
$$

$\tau$ is the transposition. In order to prove this one uses the exact sequence of functors:

$$
0 \rightarrow \Gamma^{2} \rightarrow\left(\Gamma^{1}\right)^{\otimes 2} \rightarrow\left(\Gamma^{1}\right)^{\otimes 2} \rightarrow S^{2}
$$

applied to $\mathbb{F}_{2}[u] \cong \mathrm{H}^{*}\left(V_{1}\right)$, with $\mathbb{F}_{2}[u, v] \cong \mathrm{H}^{*}\left(V_{1}\right)^{\otimes 2}$ and $\mathbb{F}_{2}\left[w_{1}, w_{2}\right] \cong$ $\Gamma^{2}\left(\mathrm{H}^{*}\left(V_{1}\right)\right)$. The morphism $\left(\Gamma^{1}\right)^{\otimes 2} \rightarrow\left(\Gamma^{1}\right)^{\otimes 2}$ being the norm map.

From the definition of $\ell^{1}$ it follows that $\ell^{1}\left(m\left(B_{2}\right)\right)=0$ and the result follows for $B_{2}$. As $B_{2}$ is the direct sum of $\bar{B}_{2}$ and $I$ result holds for $\bar{B}_{2}$.

This gives us the proof of Propositions 1.6 and 1.8 .

For the two last cases one needs to study $D_{2}$ and $\bar{D}_{2}$. It is done as follows, the subfunctor

$$
A_{2}: V \mapsto\left(\left(\mathbb{F}_{2}^{V^{\#}}\right)^{\otimes 2}\right)^{\mathbb{Z} / 3}
$$

is a direct summand in $\left.\left.\subset \mathbb{F}_{2}^{V \#}\right)^{\otimes 2} \cong \mathbb{F}_{2}^{\operatorname{Hom}\left(V, \mathbb{F}_{2}^{\oplus 2}\right)}\right)$. The action of $\mathbb{Z} / 3$ is via the inclusion into $\mathrm{GL}_{2}\left(\mathbb{F}_{2}\right)$. It is known ([17]) to be inserted in a short exact sequence:

$$
E \hookrightarrow A_{2} \rightarrow I
$$

where

$$
\bar{D}_{2} \hookrightarrow E \rightarrow \bar{D}_{2}
$$

and one has also

$$
D_{2} \hookrightarrow A_{2} \rightarrow \bar{D}_{2} \quad .
$$

in terms of unstable modules one gets corresponding sequences applying the functor $m$. As $m$ they may not be exact on the right, by inspection it is not in the first case and it is in the the two other cases. In the first case :

$$
m(E) \hookrightarrow \mathbb{F}_{2}[u, v]^{\mathbb{Z} / 3} \rightarrow \mathbb{F}_{2}[u]
$$

the image on the right being $\mathbb{F}_{2}\left[u^{2}\right]$, and

$$
\bar{D}(2) \hookrightarrow m(E) \rightarrow \bar{D}(2) \quad,
$$

and

$$
D(2) \hookrightarrow \mathbb{F}_{2}[u, v]^{\mathbb{Z} / 3} \rightarrow \bar{D}(2) \quad,
$$


$\bar{D}(2)$ being the kernel of the unique nontrivial morphism $D(2) \rightarrow \mathbb{F}_{2}[u]$. It follows that:

$$
\ell^{1}(m(E)) \cong \mathbb{F}_{2}[u] / \mathbb{F}_{2}\left[u^{2}\right] \quad,
$$

and that:

$$
\ell^{1}\left(m\left(\bar{D}_{2}\right)\right) \cong \mathbb{F}_{2}[u] / \mathbb{F}_{2}\left[u^{2}\right]
$$

In our examples we are first concerned with $D(2)$. In this case we can exploit the short exact sequence: which, as $\bar{D}(2)$ is $\mathcal{N} i l$-closed, yields immediately $\ell^{1} \circ m(D(2))=0$. This is 1.7. There remains case 1.9, keeping the notations one has a short exact sequence of unstable algebras:

$$
M_{2} \hookrightarrow D(2) \oplus H_{2} \rightarrow \mathbb{F}_{2}\left[u^{2}\right]
$$

As $\ell^{1}(D(2))=\ell^{1}\left(H_{2}\right)=0$ it impies that:

$$
\operatorname{Ext}_{\mathcal{F}}^{1}\left(\Gamma^{p-1}, \bar{D}_{2}\right) \cong \ell^{1} \circ m(\bar{D}(2))^{p-1} \cong \mathbb{F}_{2}[u] / \mathbb{F}_{2}\left[u^{2}\right]
$$

and the strong form of the conjecture is true if $p-1$ is odd.

Remark 5.6. As $\bar{D}_{2}$ is not a quotient of $\mathrm{H}^{*}\left(K_{2}\right)$, the bottom class in degree 3 , it does not appear (alone) in our list. However it is a quotient of $\mathrm{H}^{*}\left(K_{3}\right)$, thus in such a case the proof of the strong form of the conjecture would not follow because

$$
\operatorname{Ext}_{\mathcal{F}}^{1}\left(\Gamma^{p-1}, \bar{D}_{2}\right) \cong \ell^{1} \circ m(\bar{D}(2))^{p-1} \cong \mathbb{F}_{2}[u] / \mathbb{F}_{2}\left[u^{2}\right]
$$

is trivial only if $p-1$ is odd.

\section{References}

[1] John F. Adams and Clarence W. Wilkerson. Finite $H$-spaces and algebras over the Steenrod algebra. Ann. of Math. (2), 111(1):95-143, 1980 .

[2] Carlos Broto and Saïd Zarati. On sub- $\mathcal{A}_{p}^{*}$-algebras of $H^{*} V$. In Algebraic topology (San Feliu de Guixols, 1990), volume 1509 of Lecture Notes in Math., pages 35-49. Springer, Berlin, 1992.

[3] Emmanuel Dror Farjoun and J.effrey Smith. A geometric interpretation of Lannes' functor T. Number 191, pages 6, 87-95. 1990. International Conference on Homotopy Theory (Marseille-Luminy, 1988).

[4] W. G. Dwyer. Strong convergence of the Eilenberg-Moore spectral sequence. Topology, 13:255-265, 1974. 
[5] Vincent Franjou, Eric M. Friedlander, Teimuraz Pirashvili, and Lionel Schwartz. Rational representations, the Steenrod algebra and functor homology, volume 16 of Panoramas et Synthèses [Panoramas and Syntheses]. Société Mathématique de France, Paris, 2003.

[6] Vincent Franjou, Lannes Jean, and Lionel Schwartz. Autour de la cohomologie de mac lane des corps finis. Inv. math., 115:513-538, 1994.

[7] Pierre Gabriel. Des catégories abéliennes. Bull. Soc. Math. France, 90:323-448, 1962.

[8] Allen Hatcher. Algebraic topology. Cambridge University Press, Cambridge, 2002.

[9] Hans-Werner Henn, Jean Lannes, and Lionel Schwartz. The categories of unstable modules and unstable algebras over the Steenrod algebra modulo nilpotent objects. Amer. J. Math., 115(5):1053-1106, 1993.

[10] David Kraines. The $\mathcal{A}(p)$ cohomology of some $k$ stage Postnikov systems. Comment. Math. Helv., 48:56-71, 1973.

[11] Jean Lannes. Sur les espaces fonctionnels dont la source est le classifiant d'un $p$-groupe abélien élémentaire. Inst. Hautes Études Sci. Publ. Math., (75):135-244, 1992. With an appendix by Michel Zisman.

[12] Jean Lannes and Lionel Schwartz. Sur la structure des $A$-modules instables injectifs. Topology, 28(2):153-169, 1989.

[13] Jean Lannes and Lionel Schwartz. Sur les groupes d'homotopie des espaces dont la cohomologie modulo 2 est nilpotente. Israel J. Math., 66(1-3):260-273, 1989.

[14] Jean Lannes and Saïd Zarati. Sur les $\mathcal{U}$-injectifs. Ann. Sci. École Norm. Sup. (4), 19(2):303-333, 1986.

[15] John Milnor. The Steenrod algebra and its dual. Ann. of Math. (2), 67:150-171, 1958.

[16] Geoffrey M. L. Powell. The structure of indecomposable injectives in generic representation theory. Trans. Amer. Math. Soc., 350(10):41674193, 1998.

[17] Geoffrey M. L. Powell. The structure of indecomposable injectives in generic representation theory. J.P.A.A., 128:291-310, 1998.

[18] David L. Rector. Steenrod operations in the Eilenberg-Moore spectral sequence. Comment. Math. Helv., 45:540-552, 1970.

[19] Lionel Schwartz. La filtration nilpotente de la categorie $\mathcal{U}$ et la cohomologie des espaces de lacets. In Algebraic topology - rational homotopy (Louvain-la-Neuve, 1986), volume 1318 of Lecture Notes in Math., pages 208-218. Springer, Berlin, 1988. 
[20] Lionel Schwartz. Unstable modules over the Steenrod algebra and Sullivan's fixed point set conjecture. Chicago Lectures in Mathematics. University of Chicago Press, Chicago, IL, 1994.

[21] Jean-Pierre Serre. Cohomologie modulo 2 des complexes d'EilenbergMacLane. Comment. Math. Helv., 27:198-232, 1953.

[22] Larry Smith. The cohomology of stable two stage Postnikov systems. Illinois J. Math., 11:310-329, 1967.

[23] Larry Smith. Lectures on the Eilenberg-Moore spectral sequence. Lecture Notes in Mathematics, Vol. 134. Springer-Verlag, Berlin-New York, 1970 .

[24] Norman E. Steenrod. Cohomology operations. Lectures by N. E. STeenrod written and revised by D. B. A. Epstein. Annals of Mathematics Studies, No. 50. Princeton University Press, Princeton, N.J., 1962. 